\title{
The nose is the best niche for detection of pneumococcal colonisation following experimental challenge in adults of all ages
}

Elissavet Nikolaou ( $\nabla$ elissavet.nikolaou@lstmed.ac.uk )

Liverpool School of Tropical Medicine

Esther Lauryn German ( $\nabla$ esther.german@lstmed.ac.uk )

Liverpool School of Tropical Medicine

Annie Blizard

Liverpool School of Tropical Medicine

Ashleigh Howard

Liverpool School of Tropical Medicine

\section{Lisa Hitchins}

Liverpool School of Tropical Medicine

\section{Tao Chen}

Liverpool School of Tropical Medicine Jim Chadwick

Liverpool School of Tropical Medicine

Sherin Pojar

Liverpool School of Tropical Medicine

\section{Elena Mitsi}

Liverpool School of Tropical Medicine

\section{Carla Solórzano}

Liverpool School of Tropical Medicine

\section{Syba Sunny}

Royal Liverpool University Hospital

\section{Felicity Dunne}

Liverpool School of Tropical Medicine

Jenna F Gritzfeld

Liverpool School of Tropical Medicine

\section{Hugh Adler}

Liverpool School of Tropical Medicine

\section{Jason Hinds}

St George's, University of London

Katherine A Gould 
St George's, University of London

\section{Jamie Rylance}

Liverpool School of Tropical Medicine

\section{Andrea M Collins}

Liverpool School of Tropical Medicine

\section{Stephen B Gordon}

Queen Elizabeth Central Hospital

\section{Daniela M Ferreira}

Liverpool School of Tropical Medicine

\section{Research Article}

Keywords: pneumococcal niche, colonisation, Experimental Human Pneumococcal Challenge model, age

Posted Date: May 19th, 2021

DOl: https://doi.org/10.21203/rs.3.rs-513376/v1

License: (c) (i) This work is licensed under a Creative Commons Attribution 4.0 International License. Read Full License 


\section{Abstract \\ Background}

Previous studies have suggested that the pneumococcal niche changes from the nose to the oropharynx with age. We use an Experimental Human Pneumococcal Challenge model to investigate pneumococcal colonisation in each anatomical niche with age.

\section{Methods}

Healthy adults $(\mathrm{n}=112)$ were intranasally inoculated with Streptococcus pneumoniae serotype 6B (Spn6B) and were categorised as young 18-55yrs $(n=57)$ or older $>55 y r s(n=55)$. Colonisation status (frequency and density) was determined by multiplex qPCR targeting the $l y t A$ and $c p s A-6 \mathrm{~A} / \mathrm{B}$ genes in both raw and culture-enriched nasal wash and oropharyngeal swab samples collected at 2-, 7- and 14days post-exposure. For older adults, raw and culture-enriched saliva samples were also assessed.

\section{Results}

$64 \%$ of NW samples and 54\% of OPS samples were positive for Spn6B in young adults, compared to $35 \%$ of NW samples, $24 \%$ of OPS samples and $6 \%$ of saliva samples in older adults. Many colonisation events were only detected in culture-enriched samples. Experimental colonisation was detected in $72 \%$ of young adults by NW and $63 \%$ by OPS. In older adults, this was $51 \%$ by NW, $36 \%$ by OPS and $9 \%$ by saliva.

\section{Conclusions}

The nose is the best niche for detection of experimental pneumococcal colonisation in both young and older adults.

\section{Introduction}

The natural flora of the human upper respiratory tract (URT) is in constant interaction with the external environment, resulting in a diverse ecology of microorganisms colonising epithelial surfaces in the oral and nasal cavities and oropharynx ${ }^{1}$. Streptococcus pneumoniae (Spn, pneumococcus) can proliferate and establish colonisation as a non-pathogenic symbiont, which is asymptomatic for the host, particularly in adults ${ }^{2,3}$. Pneumococcus can however migrate to other organs, resulting in pneumonia, meningitis, and bacteraemia, causing significant morbidity and mortality worldwide, especially in the very young and very old.

Colonisation of the URT has been shown to be the source of, and pre-requisite for, pneumococcal disease ${ }^{4}$ and its transmission throughout the community ${ }^{5}$. Accurate detection of pneumococcal colonisation is 
crucial to assessing disease potential, as well as direct and indirect impact of vaccines. The World Health Organisation (WHO) recommends the use of nasopharyngeal swabs for pneumococcal colonisation detection in children and both nasopharyngeal and oropharyngeal swabs (OPS) in adults ${ }^{6,7}$. WHO recommendations were based on culture-based methods and more recent studies using sensitive molecular methods have encouraged other sampling methods such as saliva or nasal wash ${ }^{8,9}$, due to improved comfort and pneumococcal detection.

Many studies have investigated pneumococcal colonisation prevalence in the first few years of life, showing that the nasopharynx of $40-95 \%$ of young children ${ }^{10,11}$ is naturally colonised with pneumococcus. With the onset of adulthood, falling pneumococcal disease rates are accompanied by decreased nasopharyngeal colonisation rates at $10-25 \%{ }^{12-15}$. In older adults, a population at increased risk of pneumococcal disease, colonisation prevalence and density are reported to be lower (19), but study results are heterogeneous with some reporting very low colonisation rates of $1.9-4.2 \%{ }^{16-18}$. Our recent systematic review analysed 29 studies, 18 with participant-level data (representing 6290 participants), and reported prevalence of detected pneumococcal colonisation of $0-39 \%$ by conventional culture methods and $3-23 \%$ by molecular methods ${ }^{19}$.

Various theories have been put forward to explain the paradox of increased disease risk with decreased colonisation prevalence, including a more transient colonisation dynamic in older adults ${ }^{17}$, the poor sensitivity of conventional culture methods to detect low density colonisation in polymicrobial samples 20,21 and a transition of the pneumococcal niche from the nasopharynx to the oral cavity with ageing ${ }^{20}$.

The Experimental Human Pneumococcal Colonisation (EHPC) model is a safe, reproducible, and controlled method of studying colonisation dynamics in adult human participants, as the challenge dose and time of exposure is known ${ }^{22}$. We have recently expanded this model to older adults and reported that

experimental colonisation was established in $39 \%$ of participants $(25 / 64)$ with no adverse events. Colonisation occurred in 47\% (9/19) of participants aged 50-59 and in 42\% (13/31) of those aged 6069 compared to $21 \%(3 / 14)$ in those aged $\geq 70$ years. Colonisation density was similar between old and younger adults ${ }^{23}$.

We used 780 nasal, oropharyngeal and saliva samples to define precisely whether the niche of experimental pneumococcal colonisation changes in adults with increasing age. Association with bacterial density was also analysed.

\section{Materials And Methods}

\section{Clinical trial design, participant cohorts and sample analysis}


The methodology and inclusion/exclusion criteria for EHPC studies have been previously described ${ }^{24}$. Briefly, participants were healthy adults aged $\geq 18$ years with no major risk factors for pneumococcal disease, colonisation, or transmission (such as: cigarette smoking; close contact with children aged $<5$ years; healthcare work or caring responsibilities; steroid therapy and respiratory or immunosuppressive comorbidities). Studies were conducted in accordance with the Declaration of Helsinki and Good Clinical Practice procedures. All participants provided written informed consent and underwent a safety screening. Studies were submitted to and approved by a local NHS Research Ethics Committee. In this study, samples have been sourced from two separate EHPC clinical studies. The young vaccine study was a double-blind, randomised controlled trial conducted between September 2013 - April 2014 investigating the impact of PCV-13 vaccination on experimental pneumococcal colonisation in adults aged 18-50 (NHS REC number 12/NW/0873, ISRCTN number 45340436 registered 18/11/2013) ${ }^{25}$. In the present analysis, we include participants from the control arm only (vaccinated with Hepatitis $\mathrm{A}$ vaccine rather than PCV-13). The older adults study was an observational study of the effect of age on experimental pneumococcal colonisation in adults aged 50-80 conducted between June 2016 - February 2018 (NHS REC number 16/NW/0031, ISRCTN number 10948363 registered 08/11/2016) ${ }^{23}$. In total, 112 participants were analysed. The sample sets can be combined because the inoculation dose and strain, as well as the methods for inoculation, nasal wash collection and processing remained identical. Furthermore, pneumococcal colonisation rates and serotype distribution in healthy adults in Liverpool appears to be relatively stable, based on data collected between 2010-2017 ${ }^{26}$. The low prevalence of naturally occurring Spn6 provides reassurance that detected Spn6B is the inoculated strain. Using the full sample set, participants were split into young 18-55 years $(n=57)$ and older adults $>55$ years $(n=55)$, in keeping with the cut-off used by Marrie et al. ${ }^{27}$.

In both studies, participants were inoculated with 80,000 colony-forming units (CFU) per nostril of live Spn6B pneumococcus (BHN418, GenBank accession number ASHP00000000.1) ${ }^{25}$. Nasal wash (NW) samples were collected before inoculation to screen for pneumococcal colonisation acquired from community. Depending on the study, NW, oropharyngeal swabs (OPS) and saliva samples were then collected post pneumococcal exposure. In the young vaccine study, NW and OPS samples were collected on days 2, 7, 14 (only culture-positives) and 21 post exposure. In the older adults study, NW, OPS and saliva samples were collected on days 2, 7, 9, 14, 22 (only culture-positives) and 29 post exposure. Pneumococcal colonisation status in both studies was determined by NW culture (not discussed here) and by multiplex real-time polymerase chain reaction (qPCR) targeting the $/ y t A$ and $c p s A-6 \mathrm{~A} / \mathrm{B}$ genes in raw and culture-enriched NW, OPS, and saliva samples. The raw and culture-enriched NW, OPS, and saliva qPCR data from days 2, 7 and 14 (covered in both studies) were included in this study.

NW collection was performed as previously described ${ }^{24}$. Briefly, $20 \mathrm{~mL}$ of $0.9 \%$ sodium chloride solution in total ( $10 \mathrm{~mL}$ saline per nostril) was introduced using a syringe and held for a few seconds in the participant's nose before being expelled into a sterile container. NW was centrifuged at $4000 \mathrm{rpm}$ for 10 minutes. Supernatant was collected and pellet was resuspended in STGG (Skimmed milk-TryptoneGlucose-Glycerine) $20 \%$ glycerol before storage at $-80^{\circ} \mathrm{C}$. OPS samples were collected in $1 \mathrm{~mL}$ STGG and 
stored at $-80^{\circ} \mathrm{C}$, until further use. Saliva samples were collected using the salivette device (Sarstedt, UK). Following collection, each salivette was centrifuged at $4000 \mathrm{rpm}$ for 3 minutes at $4^{\circ} \mathrm{C}$ and after measuring the volume of saliva liquid retrieved, both pellet and supernatant were re-suspended in equal volume of STGG $50 \%$ glycerol and stored at $-80^{\circ} \mathrm{C}$, until further use.

\section{Preparation of raw and culture-enriched NW, OPS, and saliva samples}

Before DNA extraction, samples were thawed for 30 minutes at room temperature and vigorously vortexed for 20 seconds. $300 \mu \mathrm{L}$ raw NW pellet, $200 \mu \mathrm{L}$ raw OPS and $300 \mu \mathrm{L}$ raw saliva aliquots were prepared. For culture-enrichment, $50 \mu \mathrm{L}$ of NW pellet, OPS or saliva samples were plated on Columbia blood agar supplemented with $5 \%$ horse blood and $80 \mu \mathrm{L}$ gentamicin $1 \mathrm{mg} / \mathrm{mL}$. Plates were incubated overnight at $37^{\circ} \mathrm{C}$ in $5 \% \mathrm{CO}_{2}$. Following incubation, $2 \mathrm{~mL}$ of STGG $20 \%$ glycerol was added onto each plate and microbial growth scraped off. $300 \mu \mathrm{L}$ culture-enriched NW, $200 \mu \mathrm{L}$ culture-enriched OPS and 300 $\mathrm{L}$ culture-enriched saliva aliquots were prepared. Both raw and culture-enriched NW, OPS and saliva aliquots were stored at $-20^{\circ} \mathrm{C}$ until DNA extraction took place.

\section{DNA extraction on raw and culture-enriched NW, OPS, and saliva samples}

Bacterial genomic DNA was extracted from raw and culture-enriched NW, OPS, and saliva samples. On the day of the extraction, prepared aliquots were thawed at room temperature and vigorously vortexed for 20 seconds. Samples were pelleted at 20,238xg for 10 minutes. Following centrifugation, $300 \mu \mathrm{L}$ of lysis buffer with protease (Agowa Mag mini-DNA extraction kit; LGC Genomics, Germany), $100 \mu \mathrm{L}$ of zirconium beads (diameter of $0.1 \mathrm{~mm}$ ), and $300 \mu \mathrm{L}$ of phenol pH 8.0 (toxic, performed in a cabinet with charcoal filter) were added to the pellets. Samples were mechanically disrupted at $50 \mathrm{~Hz}$ for 3 minutes in a tissue homogenizer followed by 3 minutes on ice, twice. The samples were then centrifuged for 10 minutes at 9,391 $\mathrm{xg}$, and the upper aqueous phase was transferred to a sterile $1.5 \mathrm{~mL}$ Eppendorf tube pre-filled with $600 \mu \mathrm{L}$ binding buffer and $10 \mu \mathrm{L}$ magnetic beads. The samples were incubated in a mixing machine ( $265 \mathrm{rpm}$ ) for 1 hour at room temperature, then washed twice with $200 \mu \mathrm{L}$ of wash buffers 1 and 2 . Magnetic beads were dried at $55^{\circ} \mathrm{C}$ for 10 minutes, eluted in $63 \mu \mathrm{L}$ of elution buffer and stored at $-20^{\circ} \mathrm{C}$ until further use.

\section{Quantification of pneumococcal DNA by multiplex qPCR in NW, OPS, and saliva pellet samples}

We used a multiplex qPCR targeting the $l y t A^{28}$ and $c p s A-6 \mathrm{~A} / \mathrm{B}^{29}$ genes as previously described ${ }^{30}$. The reaction mixture of $25 \mu \mathrm{L}$ contained $0.6 \mu \mathrm{M}$ of each $l y t A$ primer, $0.3 \mu \mathrm{M}$ of $l y t A$ probe, $0.4 \mu \mathrm{M}$ of each $c p s A$ $6 \mathrm{~A} / \mathrm{B}$ primer, $0.2 \mu \mathrm{M}$ of $c p s A-6 \mathrm{~A} / \mathrm{B}$ probe, $12.5 \mu \mathrm{M}$ of Taqman Gene Expression Master Mix (Applied Biosystems, USA) and $2.5 \mu \mathrm{L}$ of extracted DNA. The qPCR reaction was run on a Mx3005P machine (Agilent Technologies, USA) on the following programme: 10 minutes at $95^{\circ} \mathrm{C}$ followed by 40 cycles of 15 seconds at $95^{\circ} \mathrm{C}$ and 1 minute at $60^{\circ} \mathrm{C}$. For standard curve, Spn6B DNA was extracted using the QIAamp 


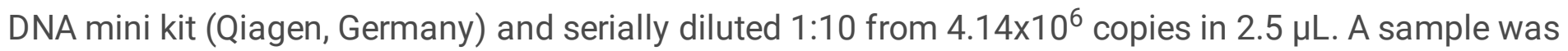
considered positive if duplicates had a CT value less than 40.

\section{Quantification and Statistical analysis}

Statistical analysis was performed by GraphPad Prism version 5.0. Contingency tables were used to assess the differences in the pneumococcal frequency in raw and culture-enriched extracted samples in both niches in each age group and in the colonisation frequency between the two niches in each age group or between the two age groups on all study days. The association was tested using Fisher's exact test and considered significant if $P<0.05$ (two-sided). Unpaired t-tests were used to compare colonisation densities in experimentally colonised participants, calculated from $c p s A-6 A / B$ gene copies in raw samples, between niches and age groups. Samples that were negative in the raw sample but positive in the corresponding culture-enriched sample were imputed a density of 1 copy/ml. A Generalized Linear Model (GLM) with binomial distribution was also used to explore the relationship between age and pneumococcal frequency in both niches.

\section{Results}

Culture-enrichment increased pneumococcal detection in all sample types in both age groups.

To detect pneumococcal presence, DNA was extracted from both raw and culture-enriched samples. Samples positive for both $l y t A$ and $c p s A-6 A / B$ genes were defined as Spn6B+, whereas those positive only for the $l y t A$ gene as $l y t A+$.

For young adults ( $n=57$, Supplementary Table S1), 147 NW samples (57 D2, 57 D7 and 33 D14) and 146 OPS samples (57 D2, 57 D7, 32 D14) were assessed. 94/147 (64\%) NW samples were Spn6B+:13 only when analysed raw, 20 only when culture-enriched, and 61 by both methods (Supplementary Table S2A: $P=0.25$ Fisher's test). 79/146 (54\%) OPS samples were Spn6B+: 45 only when culture-enriched and 34 by both methods (Supplementary Table S2B: $P<0.0001$ Fisher's test). Culture-enrichment increased the detection of SPN6B + samples in both niches with significant difference in OPS samples.

For older adults ( $\mathrm{n}=55$, Supplementary Table S1), 163 NW samples (55 D2, 55 D7 and 53 D14), 163 OPS samples (55 D2, 55 D7 and 53 D14) and 161 saliva samples (55 D2, 54 D7, 52 D14) were assessed. $57 / 163$ (35\%) NW samples were Spn6B+: 3 only when analysed raw, 20 only when culture-enriched, and 34 by both methods (Supplementary Table S3A; $P=0.0001$ Fisher's test). 39/163 (24\%) OPS samples were Spn6B+: 1 only when analysed raw, 25 only when culture-enriched, and 13 by both methods (Supplementary Table S3B: $P<0.0001$ Fisher's test). 9/161 (6\%) saliva samples were Spn6B+: 5 only when culture-enriched and 4 by both methods (Supplementary Table S3C: $P=0.0294$ Fisher's test). Culture-enrichment significantly increased the detection of Spn6B + samples in all three niches.

There was greater additional benefit in culture-enriching oropharyngeal samples (OPS and saliva) than NW in both age groups. In young adults, 21\% (20/94) and 57\% (45/79) of Spn6B + samples were detected 
by culture-enrichment only in NW and OPS respectively, indicating that culture-enrichment increased pneumococcal detection 2.7 times more in OPS samples than NW. In older adults, $35 \%$ (20/57), 64\% $(25 / 39)$ and $56 \%(5 / 9)$ of Spn6B + samples were detected by culture-enrichment only in NW, OPS and saliva respectively, indicating that culture-enrichment increased pneumococcal detection 1.1-1.8 times more in oropharyngeal (OPS and saliva) samples than in NW. Moreover, in case of the oropharyngeal niche, pneumococcal detection rates in OPS were 1.6 times higher than in saliva.

Pneumococcal colonisation frequency and density with ageing in both nose and oropharynx.

To investigate the kinetics of experimental colonisation in both niches, we assessed the colonisation frequency and density of pneumococcal DNA in both age groups on days 2, 7 and 14 post pneumococcal exposure as shown in Fig. 1. For D14, only data from culture-positive participants in the older adults study was analysed to ensure comparability with the young vaccine study data. Pneumococcal colonisation frequency was significantly higher in young than older adults at all study days post exposure (Fig. 1A) in both NW (Young vs Older: Day $236 / 57$ (63\%) vs 22/55 (40\%), $P=0.014$, Day 7 $34 / 57(60 \%)$ vs $19 / 55$ (34.5\%), $P=0.008$ and Day $1423 / 29$ (79.3\%) vs $11 / 19(57.9 \%), P=0.1931)$ and OPS (Young vs Older: Day $228 / 57$ (49.1\%) vs 10/55 (18.2\%), $P=0.0007$, Day $729 / 57$ (50.9\%) vs 14/55 (25.5\%), $P=0.007$ and Day $1422 / 28$ (78.6\%) vs 11/19 (57.9\%), $P=0.1946)$. Comparing the two niches in both age groups separately, pneumococcal frequency was higher in the nose than the oropharynx at days 2 and 7 and similar at day 14 post pneumococcal exposure in both age groups (Fig. 1A, older adults NW $22 / 55(40 \%)$ vs OPS $10 / 55(18.2 \%)$ D $2, P=0.020)$.

Pneumococcal colonisation density was higher in young adults than in older adults for OPS $(P=0.008)$ but not for NW $(P=0.30)$ (Fig. 1B). Pneumococcal colonisation density was higher in NW than in OPS for older adults $(P=0.016)$ but not for young adults $(P=0.09)$ (Fig. 1B).

Pneumococcal presence is higher in the nose (NW) than oropharynx (OPS) within and between young and older adults.

In order to compare overall pneumococcal detection rates between the nasal and oropharyngeal niches, an overall nasal (combined raw and culture-enriched NW) and oropharyngeal (combined raw and cultureenriched OPS) profile were created for each participant and plotted on a heat map as shown in Fig. 2. When extraction from raw and CE samples yielded different results, the positive result was retained regardless of the method. Participants with qPCR-negative samples on all study days were defined as negative (shown in white). Those with a Spn6B + sample on any study day were classified as experimentally colonised (shown in black). Participants with a lyt $A+$ but $c p s A-6 A / B$ - sample on any study day were classified as colonised with a lytA-carrying streptococcus (shown in grey). Participants with Spn6B + samples and lytA+ (cpsA-6A/B -) samples on different study days were classified as cocolonised (shown with hatched shading).

The nose of 41/57 (72\%) young adults was colonised with Spn6B (Fig. 2A). Using NW, 12/57 (21\%) participants were negative, $37 / 57(65 \%)$ experimentally colonised, $4 / 57(7 \%)$ colonised with a lytA- 
carrying streptococcus and 4/57 (7\%) co-colonised. The oropharynx of 36/57 (63\%) young adults was colonised with Spn6B (Fig. 2A). Using OPS, 16/57 (28\%) participants were negative, 29/57 (51\%) experimentally colonised, 5/57 (9\%) colonised with a lytA-carrying streptococcus and 7/57 (12\%) cocolonised.

The carriage rate in older adults was lower than in younger adults. The nose of $28 / 55(51 \%)$ older adults was colonised with Spn6B (Fig. 2B). Using NW, 22/55 (40\%) participants were negative, 24/55 (44\%) experimentally colonised, $5 / 55,(9 \%)$ colonised with a lytA-carrying streptococcus and $4 / 55(7 \%)$ cocolonised. The oropharynx of 20/55 (36\%) older adults, as assessed by OPS, was colonised with Spn6B (Fig. 2B). Using OPS, 22/55 (40\%) participants were negative, 19/55 (35\%) experimentally colonised, $12 / 55(24 \%)$ colonised with a lytA-carrying streptococcus and 1/55 (2\%) co-colonised. Because there were more participants colonised with lytA-carrying streptococci in their oropharynx in the older cohort, we analysed these samples by microarray. In all 12 cases, non-pneumococcal streptococci were identified (data not shown).

Using combined raw and culture-enrichment methods, higher pneumococcal presence was detected in the nose than the oropharynx in both age groups with statistical significance in older adults (Supplementary Table S4, $P=0.016)$. Pneumococcal presence was significantly different between young and older adults in both NW (Supplementary Table S4, $P=0.026$ ) and OPS (Supplementary Table S4, $P=0.004$ ).

OPS is more sensitive than saliva for pneumococcal detection in older adults.

Both OPS (described above, combined raw and culture-enriched OPS) and saliva (combined raw and culture-enriched saliva) samples were used to assess pneumococcal colonisation in the oropharynx of older adults. In saliva, 36/55 (65\%) participants were negative, 5/55 (9\%) experimentally colonised, 14/55 (25\%) colonised with a /ytA-carrying streptococcus and no co-colonised were detected. Therefore, overall, the oropharynx of $20 / 55(36 \%)$ older adults, as assessed by both OPS and saliva, were colonised with Spn6B (Fig. 2B). Only 5 of these (25\%) were detected in saliva compared to 19 (95\%) in OPS, (Fig. 2B), indicating that in the older age group, saliva is a less sensitive method of assessing pneumococcal colonisation than OPS.

\section{Discussion}

This study investigated whether the pneumococcal colonisation niche alters with increasing age following experimental human challenge. Our findings show that regardless of age, the nasal niche had the highest percentage of experimentally colonised participants.

Experimental colonisation involves the direct inoculation of pneumococcus into the nose and therefore may not precisely imitate natural colonisation dynamics. Nevertheless, we have described in a series of independent studies that participants who become colonised following inoculation develop a consistent colonisation episode of $1-3$ weeks of similar density to natural colonisation ${ }^{24,31}$. Our findings agree with 
studies of natural colonisation where a higher incidence of pneumococcal growth is found in individuals' nasopharyngeal samples compared with their oropharyngeal samples ${ }^{15,32}$.

Young adults showed a higher percentage of colonised participants than their older counterparts at each study day following inoculation, in both the nose and the oropharynx. The relationship between age and prevalence of colonisation has been well documented ${ }^{33-35}$, in agreement with our findings that prevalence of colonisation decreases with age. This decrease did not reach statistical significance when samples were analysed using classical microbiology methods $(P=0.19)$, in keeping with the increased sensitivity of molecular methods.

Culture-enrichment of samples increased pneumococcal detection in both niches in both age groups. This extra step has been shown previously to increase pneumococcal detection in saliva ${ }^{20,36}$ and our group now uses it routinely when analysing clinical trial samples. Although labour-intensive, we believe that its added value justifies recommending it as standard practice in combination with analysing raw samples ${ }^{37}$.

The strength of our study is the collection of paired longitudinal nasal and oropharyngeal samples before and after pneumococcal inoculation of a known strain. Coupled with culture enrichment and molecular methods for capsular polysaccharide-specific detection, this allows the precise determination of the frequency and density of bacteria for each study day and niche, according to age. A weakness of our work is the lack of saliva in the young cohort and that collection of samples in the older and young cohorts was conducted during different studies. However, the strain used for inoculation as well as the methods for inoculation, nasal wash collection and processing remained identical, allowing for direct comparison of cohorts as done previously ${ }^{23}$. A further limitation could extend to the methods used for nasal sampling and saliva collection. Nasal wash is more comfortable and more sensitive than nasopharyngeal swab for pneumococcal detection in adults ${ }^{9}$, however it is not always feasible outside of a clinic setting. It may be that we would not have seen such a difference between the nose and oropharynx if we had used nasopharyngeal swabs. Our saliva detection levels were also much lower than those reported elsewhere when a spitting method was used for sample collection ${ }^{20,37}$. In older adults, with drier mouths, low sample volumes could be obscured by the use of the Salivette device.

Our results indicate age-related host factors could affect colonisation prevalence in these age groups. The percentage of participants showing experimental carriage fell between days 2 and day 14 in the nose of older age group, as assessed by NW. This could be evidence of pneumococcal colonisation clearance, which is influenced by host immune responses such as local phagocytic function and acute mucosal inflammatory responses ${ }^{37}$ as well as mechanisms known to be affected by immunosenescence (toll like receptors and reduction in the function of host signalling pathways) ${ }^{38}$. Unlike pneumococcal colonisation prevalence, pneumococcal density in NW was unaffected by sampling age. Several studies have found an opposite relationship between nasopharyngeal colonisation density and age indicating higher pneumococcal densities in younger subjects ${ }^{39-41}$. 
It has been established that the complexity and diversity of the microbiome increases from the nasopharynx to the oropharynx to saliva ${ }^{42}$. Investigation of NW, OPS and saliva samples in this study showed a higher percentage of participants colonised with a lytA-carrying streptococcus in OPS (and saliva in older adults) in contrast to NW in both age groups. Due to higher levels of species diversity within the oral cavity, and the capacity of pneumococci to exchange genes with other streptococci, the use of lytA as a PCR target needs to be treated with caution. Positive lytA PCR results may indicate the presence of non-pneumococcal species in addition to pneumococcal species, leading to false positives, which was indeed the case here. In addition, in our experience the addition of saliva sampling in older adults was not beneficial as it is less sensitive when compared with OPS.

In summary, this study has shown that the optimal sampling niche to detect experimental pneumococcal colonisation is the nose regardless of age, as assessed by NW. However, individuals show different colonised niches, so reducing sampling to only the nose would exclude detection of pneumococcal colonisation in some patients (both young and older adults). Future studies could investigate the site of pneumococcal colonisation over a longer time-period following experimental inoculation. The current study examined a short period of time, and studies in the literature of natural carriage are snapshots in time.

\section{Declarations}

\section{Funding}

This work was supported by the Medical Research Council/FAPESP (grant number MR/M011569/1) and the Bill \& Melinda Gates Foundation (grant number OPP1117728).

\section{Acknowledgements}

We would like to thank all participants for their participation.

\section{Author contributions}

JR, AMC, HA, SBG and DMF designed and supervised the two clinical trials; EN, ELG, SP, EM and JFG processed clinical trial samples; EN, ELG, CSG and EM designed and optimised the multiplex qPCR; EN, ELG, AB, AH, LH, SS, FD and JFG performed DNA extractions and qPCRs; JH and KAG performed microarray analysis; EN, ELG and JC performed data analysis; TC performed statistical analysis; EN and ELG prepared the manuscript text, tables and figures; All authors approved the manuscript.

\section{Data availability}

The datasets generated during and/or analysed during the current study are available from the corresponding author on reasonable request.

\section{Additional Information}


The authors declare no competing interests.

\section{References}

1. Costello, E. K., Stagaman, K., Dethlefsen, L., Bohannan, B. J. M. \& Relman, D. A. The application of ecological theory toward an understanding of the human microbiome. Science. 336, 1255-1262 https://doi.org/10.1126/science.1224203 (2012).

2. Hales, C. et al. Symptoms associated with influenza vaccination and experimental human pneumococcal colonisation of the nasopharynx. Vaccine. 38, 2298-2306 https://doi.org/10.1016/j.vaccine.2020.01.070 (2020).

3. Trimble, A. et al. Pneumococcal colonisation is an asymptomatic event in healthy adults using an experimental human colonisation model. PLoS One. 15, e0229558 https://doi.org/10.1371/journal.pone.0229558 (2020).

4. Simell, B. et al. The fundamental link between pneumococcal carriage and disease. Expert Review of Vaccines. 11, 841-855 https://doi.org/10.1586/erv.12.53 (2012).

5. Melegaro, A., Gay, N. J. \& Medley, G. F. Estimating the transmission parameters of pneumococcal carriage in households. Epidemiology and infection. 132, 433-441 https://doi.org/10.1017/s0950268804001980 (2004).

6. O’Brien, K. L., Nohynek, H. \& The Who Pneumococcal Vaccine Trials Carriage Working. \&, G. Report from a WHO Working Group: standard method for detecting upper respiratory carriage of Streptococcus pneumoniae. Pediatr Infect Dis J22 (2003).

7. Satzke, C. et al. Standard method for detecting upper respiratory carriage of Streptococcus pneumoniae: Updated recommendations from the World Health Organization Pneumococcal Carriage Working Group. Vaccine. 32, 165-179 https://doi.org/10.1016/j.vaccine.2013.08.062 (2013).

8. Arguedas, A. et al. Upper respiratory tract colonization with Streptococcus pneumoniae in adults. Expert Review of Vaccines. 19, 353-366 https://doi.org/10.1080/14760584.2020.1750378 (2020).

9. Gritzfeld, J. F., Roberts, P., Roche, L., El Batrawy, S. \& Gordon, S. B. Comparison between nasopharyngeal swab and nasal wash, using culture and PCR, in the detection of potential respiratory pathogens. BMC research notes. 4, 122 https://doi.org/10.1186/1756-0500-4-122 (2011).

10. Bomar, L., Brugger, S. D. \& Lemon, K. P. Bacterial microbiota of the nasal passages across the span of human life. Curr Opin Microbiol. 41, 8-14 https://doi.org/10.1016/j.mib.2017.10.023 (2018).

11. Murad, C. et al. Pneumococcal carriage, density, and co-colonization dynamics: A longitudinal study in Indonesian infants. International Journal of Infectious Diseases. 86, 73-81 https://doi.org/10.1016/j.ijid.2019.06.024 (2019).

12. Goldblatt, D. et al. Antibody responses to nasopharyngeal carriage of Streptococcus pneumoniae in adults: a longitudinal household study. The Journal of infectious diseases. 192, 387-393 https://doi.org/10.1086/431524 (2005). 
13. Heinsbroek, E. et al. Persisting high prevalence of pneumococcal carriage among HIV-infected adults receiving antiretroviral therapy in Malawi: a cohort study. AIDS. 29, 1837-1844 https://doi.org/10.1097/QAD.0000000000000755 (2015).

14. Numminen, E. et al. Climate induces seasonality in pneumococcal transmission. Sci Rep. 5, 1134411344 https://doi.org/10.1038/srep11344 (2015).

15. Watt, J. P. et al. Nasopharyngeal versus oropharyngeal sampling for detection of pneumococcal carriage in adults. Journal of clinical microbiology. 42, 4974-4976 https://doi.org/10.1128/jcm.42.11.4974-4976.2004 (2004).

16. Becker-Dreps, S. et al. Pneumococcal Carriage and Vaccine Coverage in Retirement Community Residents. Journal of the American Geriatrics Society. 63, 2094-2098 https://doi.org/10.1111/jgs.13651 (2015).

17. Flamaing, J., Peetermans, W. E., Vandeven, J. \& Verhaegen, J. PNEUMOCOCCAL COLONIZATION IN OLDER PERSONS IN A NONOUTBREAK SETTING. Journal of the American Geriatrics Society. 58, 396-398 https://doi.org/10.1111/j.1532-5415.2009.02700.x (2010).

18. Zanella, R. C. et al. Nasopharyngeal carriage of Streptococcus pneumoniae, Haemophilus influenzae, and Staphylococcus aureus in a Brazilian elderly cohort. PLOS ONE. 14, e0221525 https://doi.org/10.1371/journal.pone.0221525 (2019).

19. Smith, E. L. et al. Upper airways colonisation of Streptococcus pneumoniae in adults aged 60 years and older: A systematic review of prevalence and individual participant data meta-analysis of risk factors. The Journal of infection. 81, 540-548 https://doi.org/10.1016/j.jinf.2020.06.028 (2020).

20. Krone, C. L. et al. Carriage of Streptococcus pneumoniae in Aged Adults with Influenza-Like-Illness. PLOS ONE. 10, e0119875 https://doi.org/10.1371/journal.pone.0119875 (2015).

21. van Deursen, A. M., van den Bergh, M. R. \& Sanders, E. A. Carriage of Streptococcus pneumoniae in asymptomatic, community-dwelling elderly in the Netherlands. Vaccine. 34, 4-6 https://doi.org/10.1016/j.vaccine.2015.11.014 (2016).

22. Gritzfeld, J. F. et al. Experimental human pneumococcal carriage. Journal of visualized experiments: JoVE. https://doi.org/10.3791/50115 (2013).

23. Adler, H. et al. Experimental Human Pneumococcal Colonisation in Older Adults is Feasible and Safe, Not Immunogenic. Am J Respir Crit Care Med. https://doi.org/10.1164/rccm.202004-14830C (2020).

24. Ferreira, D. M. et al. Controlled human infection and rechallenge with Streptococcus pneumoniae reveals the protective efficacy of carriage in healthy adults. Am J Respir Crit Care Med. 187, 855-864 https://doi.org/10.1164/rccm.201212-22770C (2013).

25. Collins, A. M. et al. First Human Challenge Testing of a Pneumococcal Vaccine. Double-Blind Randomized Controlled Trial. Am J Respir Crit Care Med. 192, 853-858 https://doi.org/10.1164/rccm.201503-05420C (2015).

26. Adler, H. et al. Pneumococcal Colonization in Healthy Adult Research Participants in the Conjugate Vaccine Era, United Kingdom, 2010-2017. The Journal of infectious diseases. 219, 1989-1993 https://doi.org/10.1093/infdis/jiz034 (2019). 
27. Marrie, T. J., Tyrrell, G. J., Majumdar, S. R. \& Eurich, D. T. Effect of Age on the Manifestations and Outcomes of Invasive Pneumococcal Disease in Adults. The American journal of medicine 131, 100.e. (2018).

28. Carvalho Mda, G. et al. Evaluation and improvement of real-time PCR assays targeting lytA, ply, and psaA genes for detection of pneumococcal DNA. Journal of clinical microbiology. 45, 2460-2466 https://doi.org/10.1128/jcm.02498-06 (2007).

29. Azzari, C. et al. Realtime PCR is more sensitive than multiplex PCR for diagnosis and serotyping in children with culture negative pneumococcal invasive disease. PloS one. 5, e9282-e9282 https://doi.org/10.1371/journal.pone.0009282 (2010).

30. German, E. L. et al. Protective effect of PCV vaccine against experimental pneumococcal challenge in adults is primarily mediated by controlling colonisation density. Vaccine. 37, 3953-3956 https://doi.org/10.1016/j.vaccine.2019.05.080 (2019).

31. Gritzfeld, J. F. et al. Density and duration of experimental human pneumococcal carriage. Clinical microbiology and infection: the official publication of the European Society of Clinical Microbiology and Infectious Diseases. 20, 01145-1151 https://doi.org/10.1111/1469-0691.12752 (2014).

32. Lieberman, D. et al. Nasopharyngeal versus oropharyngeal sampling for isolation of potential respiratory pathogens in adults. Journal of clinical microbiology. 44, 525-528 https://doi.org/10.1128/jcm.44.2.525-528.2006 (2006).

33. Grant, L. R. et al. Impact of the 13-Valent Pneumococcal Conjugate Vaccine on Pneumococcal Carriage Among American Indians. Pediatr Infect Dis J. 35, 907-914 https://doi.org/10.1097/INF.0000000000001207 (2016).

34. Mackenzie, G. A., Leach, A. J., Carapetis, J. R., Fisher, J. \& Morris, P. S. Epidemiology of nasopharyngeal carriage of respiratory bacterial pathogens in children and adults: cross-sectional surveys in a population with high rates of pneumococcal disease. BMC Infectious Diseases. 10, 304 https://doi.org/10.1186/1471-2334-10-304 (2010).

35. Scott, J. R. et al. Impact of more than a decade of pneumococcal conjugate vaccine use on carriage and invasive potential in Native American communities. The Journal of infectious diseases. 205, 280-288 https://doi.org/10.1093/infdis/jir730 (2012).

36. Wyllie, A. L. et al. Molecular surveillance on Streptococcus pneumoniae carriage in non-elderly adults; little evidence for pneumococcal circulation independent from the reservoir in children. Sci Rep. 6, 34888 https://doi.org/10.1038/srep34888 (2016).

37. Nikolaou, E. et al. Experimental Human Challenge Defines Distinct Pneumococcal Kinetic Profiles and Mucosal Responses between Colonized and Non-Colonized Adults. mBio 12, doi:10.1128/mBio.02020-20 (2021).

38. Krone, C. L., van de Groep, K., Trzciński, K., Sanders, E. A. \& Bogaert, D. Immunosenescence and pneumococcal disease: an imbalance in host-pathogen interactions. The Lancet. Respiratory medicine. 2, 141-153 https://doi.org/10.1016/s2213-2600(13)70165-6 (2014). 
39. Roca, A. et al. Effect of age and vaccination with a pneumococcal conjugate vaccine on the density of pneumococcal nasopharyngeal carriage. Clin Infect Dis. 55, 816-824 https://doi.org/10.1093/cid/cis554 (2012).

40. Trzciński, K. et al. Superiority of trans-oral over trans-nasal sampling in detecting Streptococcus pneumoniae colonization in adults. PloS one. 8, e60520-e60520 https://doi.org/10.1371/journal.pone.0060520 (2013).

41. Sutcliffe, C. G. et al. Association of Laboratory Methods, Colonization Density, and Age With Detection of Streptococcus pneumoniae in the Nasopharynx. American journal of epidemiology. 188, 2110-2119 https://doi.org/10.1093/aje/kwz191 (2019).

42. Tavares, D. A. et al. Identification of Streptococcus pneumoniae by a real-time PCR assay targeting SP2020. Sci Rep. 9, 3285 https://doi.org/10.1038/s41598-019-39791-1 (2019).

\section{Figures}


A

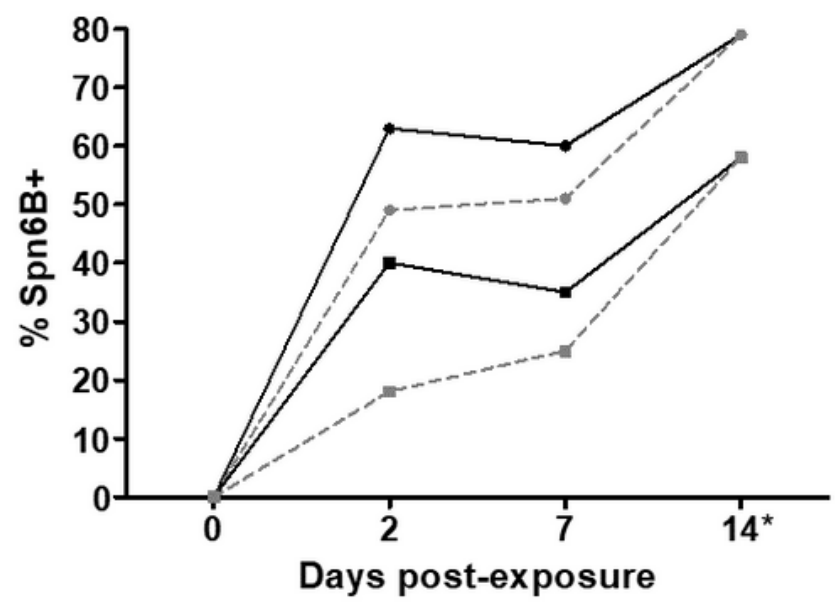

$\rightarrow$ NW Young n: D2=36/57, D7=34/57, D14=23/29

- OPS Young n: D2=28/57, D7=29/57, D14=22/28

- NW Older n: D2=22/55, D7=19/55, D14=11/19

-. OPS Older n: D2=10/55, D7=14/55, D14=11/19

* culture-positive participants only

B

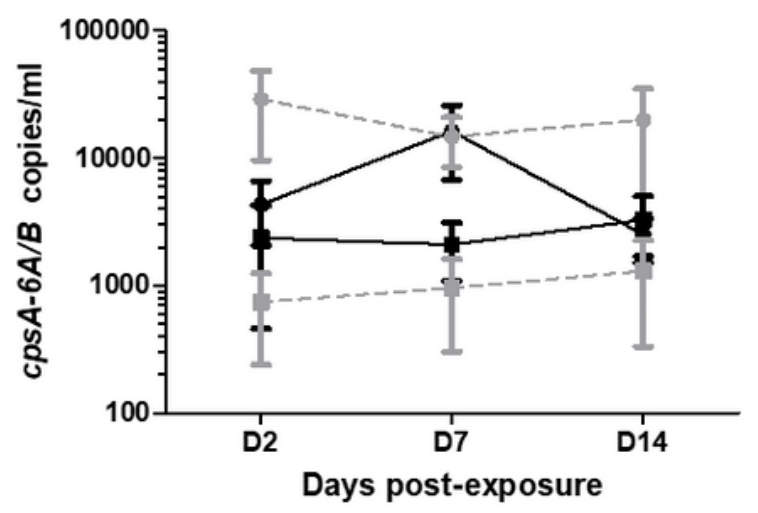

$\rightarrow$ Young NW

- Young OPS

- Older NW

-1. Older OPS

\section{Figure 1}

Experimental pneumococcal colonization in young (18-55yrs, $n=57)$ and older adults ( $>55 y r s, n=55)$. A. Frequency of Spn6B pneumococcus in nose (NW) and oropharynx (OPS) in both age groups. Detection of pneumococcal DNA was determined by multiplex qPCR. Participants with qPCR results positive for Spn6B $(C T<40)$ in the nose and oropharynx per day post-exposure per age group were: Young NW: D2 $n=36 / 57$, D7 n=34/57, D14 n=23/29, Young OPS: D2 n=28/57, D7 n=29/57, D14 n=22/28, Older NW: D2 
$n=22 / 55, D 7 n=19 / 55, D 14 n=11 / 19$, Older OPS: D2 n=10/55, D7 n=14/55, D14 n=11/19. The number of volunteers with Spn6B+ sample (CT <40) in each day post-exposure is expressed as a percentage (\%) of the total number of volunteers. Statistical significance was assessed by Fisher's contingency test: Young vs Older adults: NW D2 P=0.014, D7 P=0.008, D14 P=0.1931 and OPS D2 P=0.0007, D7 P=0.007, D14 $P=0.1946$, Older adults NW vs OPS: $D 2 * P=0.020$. B. Density of Spn6B pneumococcus in nose $(N W)$ and oropharynx (OPS) in both age groups. Each time point represents the average density of SPN6B+ per study day per niche. Data was log transformed.
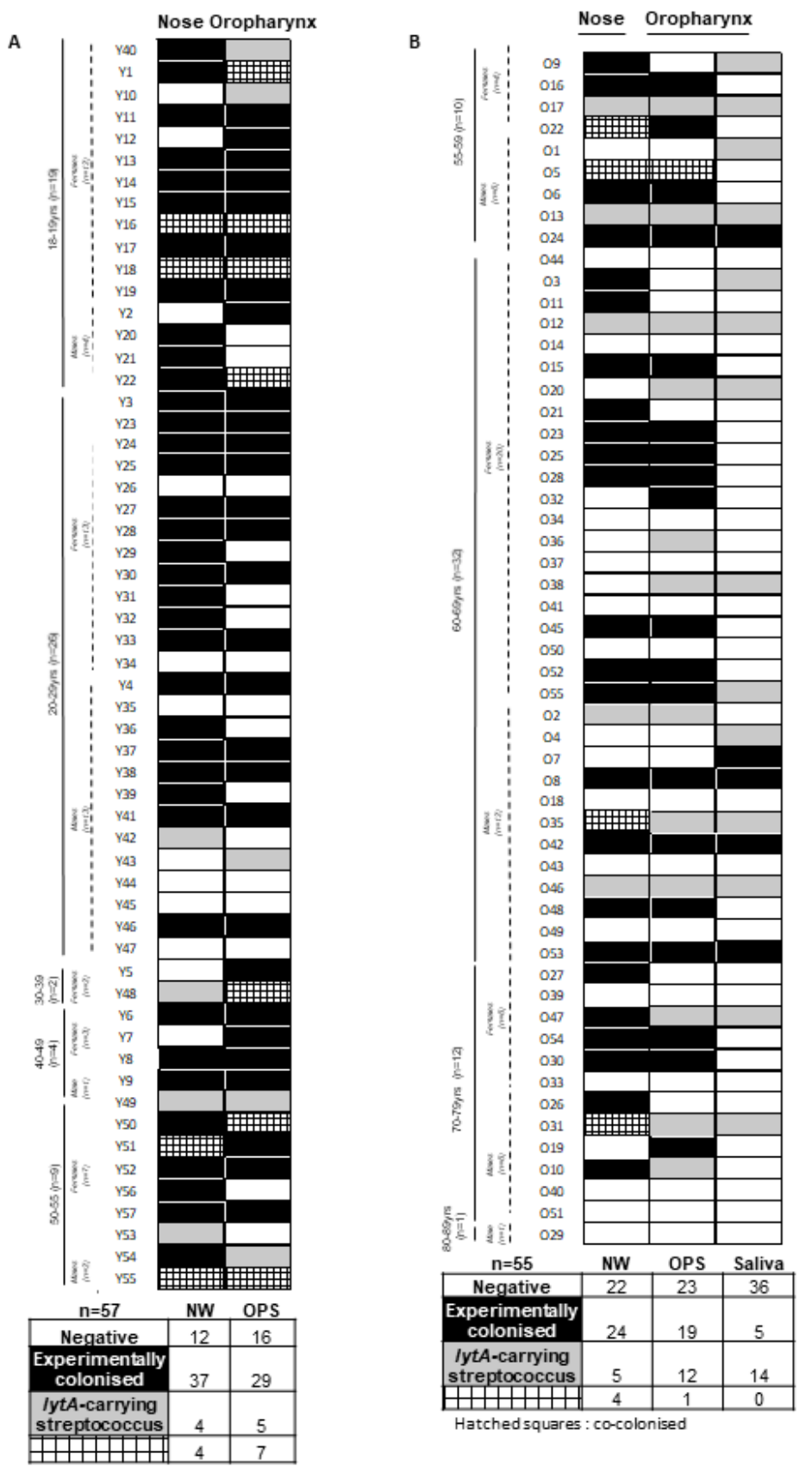

Hatched squares : co-colonised 


\section{Figure 2}

Heatmap showing individual nasal and oropharyngeal profiles in A. young (18-55yrs, $n=57)$ and $B$. older $(>55 y r s, n=55)$ adults. Participants are colour coded; white squares qPCR-negative, black squares $\mathrm{Spn} 6 \mathrm{~B}+$, grey squares lytA+ and hatched squares co-colonisers. Data is broken down by age group and gender. More Spn6B+ participants were detected in nose (NW) than oropharynx (OPS) in both age groups (Young: NW $n=41$ vs OPS $n=36$, Older: NW $n=28$ vs OPS $n=20$ ). Pneumococcal presence in both NW (Young $n=41$ vs Older $n=28,{ }^{*} P=0.026$ ) and OPS (Young $n=36$ vs Older $n=20,{ }^{*} P=0.008$ ) was statistically significantly different between young and older adults (GLM model). In older adults, fewer Spn6B+ participants were detected in saliva $(n=5)$.

\section{Supplementary Files}

This is a list of supplementary files associated with this preprint. Click to download.

- SupplementaryTables.docx 